\title{
Low dose DTIC is effective and safe in pretreated patients with well differentiated neuroendocrine tumors
}

\author{
Daniela Mueller ${ }^{1}$, Sebastian Krug${ }^{2}$, Moushumee Majumder ${ }^{1}$, Anja Rinke ${ }^{1 * \dagger}$ and Thomas Matthias Gress ${ }^{1+}$
}

\begin{abstract}
Background: Streptozocin (STZ) based chemotherapy is recommended for patients with metastatic pancreatic neuroendocrine tumors (pNET). Temozolomide as mono- or combination therapy has been suggested to be a promising alternative. However, the treatment is costly and not approved for the treatment of pNETs. Dacarbazine (DTIC) shares the active metabolite with temozolomide and is broadly available at a low cost. The aim of this study was a retrospective evaluation of the efficacy and tolerability of a lower dose DTIC-regimen in patients with progressive advanced NETs.

Methods: We retrospectively analyzed 75 patients with NETs predominantly of pancreatic origin treated at our center between 1998 and 2013. 650 mg/m² of DTIC were administered intravenously over 60 min every 4 weeks. Morphological response was assessed according to RECIST1.1 criteria. The median progression free survival (PFS) was calculated using Kaplan-Meier and Cox regression methods, respectively. Univariate analyses of possible prognostic markers were performed.
\end{abstract}

Results: The objective response rate (ORR) was $27 \%$ for the entire cohort and $32 \%$ in 50 pNET patients, respectively. Stable disease (SD) was documented in 29 patients (39\%). Median PFS (mPFS) in patients receiving DTIC was 7 months (3.9-10; $95 \%$ confidence interval). Radiological and biochemical response were the only significant prognostic markers for longer PFS in univariate analysis. Treatment was well tolerated. Nausea was the most common side effect (31 \%), only one case (1.3\%) of grade 3 toxicity (vomiting) occurred.

Conclusion: Low dose DTIC chemotherapy is an effective and well-tolerated treatment option in patients with progressive well differentiated neuroendocrine neoplasms, especially of pancreatic origin.

Keywords: Dacarbazine, Neuroendocrine tumor, Chemotherapy, Objective response, Prognostic markers

\section{Background}

With the advent of novel molecular targeted treatments such as everolimus and sunitinib the therapeutic armamentarium in metastatic neuroendocrine tumors (NET) has broadened. Chemotherapy is still considered first line treatment in specific patient subgroups such as patients with neuroendocrine carcinoma where platinumbased chemotherapy is recommended [1]. The European Neuroendocrine Tumor Society (ENETS) suggests using

\footnotetext{
* Correspondence: sprengea@uni-marburg.de

${ }^{\dagger}$ Equal contributors

${ }^{1}$ Department of Gastroenterology, University Hospital Marburg, Baldinger

Strasse, D35043 Marburg, Germany

Full list of author information is available at the end of the article
}

a streptozocin-based chemotherapy as first line treatment in metastatic pancreatic neuroendocrine tumors (pNET) G1 and G2 with a high tumor burden or tumorrelated local symptoms [2] and as second line treatment in progressive pNET. In recent years some small studies reported promising results for the alkylating agent temozolomide as single- or combination therapy. In a retrospective study first-line treatment of 30 patients with pancreatic neuroendocrine tumors (pNET) with temozolomide and capecitabine resulted in an impressive response rate of $70 \%$ and a 2 year survival rate of $92 \%$ [3]. Dacarbazine is an alkylating agent sharing the active metabolite metozolomide with temozolomide. Different regimens of dacarbazine have been used for more than 
three decades. Early reports included clinical and morphological responses in patients with glucagonoma syndrome [4, 5]. The largest monotherapy study so far comprised 50 patients with progressive pNET treated with $850 \mathrm{mg} / \mathrm{m} 2$ dacarbazine every 4 weeks. The response rate of this Eastern Cooperative Oncology Group study was $33 \%$. Most responders had not received prior chemotherapies [6]. This protocol was associated with relevant toxicities including two deaths, $13 \%$ grade 3 vomiting and $10 \%$ grade 4 hematotoxicity. In another large randomized trial of the Eastern Cooperative Oncology Group dacarbazine as second line treatment in patients with carcinoid tumors resulted in a response rate of only $8.2 \%$ [7].

In this study we retrospectively investigated the efficacy and safety of a modified, less dose intense dacarbazine treatment schedule comprising single intravenous applications of $650 \mathrm{mg} / \mathrm{m}^{2}$ DTIC every 4 weeks in a cohort of 75 mostly pretreated patients with well differentiated neuroendocrine neoplasms consecutively treated at our institution.

\section{Methods}

\section{Patients}

We retrospectively evaluated 75 consecutive patients with histologically confirmed well differentiated neuroendocrine neoplasms who were treated at our hospital with dacarbazine between 1998 and 2013. All patients had measurable disease according to the Response Evaluation Criteria in Solid Tumors (RECIST 1.1). A total of 40 male and 35 female patients with a median age of 56 years (range 28-80) were treated. Patient characteristics are summarized in Table 1.

For all but four patients dacarbazine represented at least the second line of treatment, median number of pretreatments was two $(0-5)$. Thus the cohort is representative for heavily pretreated patients (details of the pretreatments are given as Additional file 1: Table S1).

\section{Treatment and evaluation}

DTIC treatment was initiated after documented tumor progression. $650 \mathrm{mg} / \mathrm{m} 2$ of DTIC were administered intravenously over $60 \mathrm{~min}$ every 4 weeks. All patients received granisetron as antiemetic premedication.

Patients were restaged every 3 months using CT or MRI-scans and by measuring serum chromogranin A $(\mathrm{CgA})$ levels. Biochemical response was defined as a decrease of CgA of at least $30 \%$. Morphological response was assessed according to RECIST1.1 criteria. Side effects were collected from the medical files and classified according to the common toxicity grading system (Common Terminology Criteria for Adverse Events Version 4.0).
Table 1 Summary of patient characteristics

\begin{tabular}{|c|c|c|}
\hline Characteristic & Number & Percent \\
\hline Age (years) & median: 56 & range: $27-78$ \\
\hline$<60$ & 49 & 65.3 \\
\hline$>60$ & 26 & 34.7 \\
\hline \multicolumn{3}{|l|}{ Sex } \\
\hline Male & 40 & 53 \\
\hline Female & 35 & 47 \\
\hline \multicolumn{3}{|l|}{ Localization of primary } \\
\hline Pancreas & 50 & 66.6 \\
\hline Midgut & 11 & 14.6 \\
\hline Bronchus & 6 & 8 \\
\hline Stomach & 1 & 1.3 \\
\hline Hindgut & 2 & 2.6 \\
\hline Thymus & 1 & 1.3 \\
\hline Unknown & 4 & 5.3 \\
\hline \multicolumn{3}{|l|}{ Tumor grading } \\
\hline G1 & 27 & 36 \\
\hline G2 & 34 & 45 \\
\hline Unspecified & 14 & 19 \\
\hline \multicolumn{3}{|l|}{ Prior treatment } \\
\hline 1 prior treatment & 24 & 32 \\
\hline$>1$ prior treatment & 43 & 57 \\
\hline No pretreatment & 4 & 5.3 \\
\hline No data available & 4 & 5.3 \\
\hline \multicolumn{3}{|l|}{ Metastases } \\
\hline No metastases (locally advanced) & 2 & 2.6 \\
\hline Liver only & 33 & 44 \\
\hline $\begin{array}{l}\text { Liver plus additional } \\
\text { Extrahepatic metastases } \\
\text { (Bone, lung, spleen) }\end{array}$ & 40 & 53 \\
\hline
\end{tabular}

\section{Statistical analysis}

The median progression free survival (PFS) was calculated using Kaplan-Meier and Cox regression methods, respectively. Univariate analyses were performed. All statistical calculations were performed using SPSS (IBM SPSS Statistics). Differences were considered statistically significant when the $\mathrm{P}$ value was less than 0.05 . The primary endpoint was the objective response rate (ORR). Secondary endpoints included progression free survival (time from first dose of chemotherapy until documentation of tumor progression or death), duration of response and toxicity.

\section{Results}

Efficacy

A median of eight courses of DTIC (range 3-46) were administered. A biochemical response was observed in 19 of 39 patients (49\%) with elevated plasma CgA 
before dacarbazine therapy. Median PFS (mPFS) in patients receiving DTIC was 7 months $(3.9-10$; $95 \%$ confidence interval). A partial remission (PR) could be documented in 20/75 patients (27\%). Responding patients had well differentiated NETs of pancreatic $(n=$ 16), intestinal $(n=1)$, bronchial $(n=2)$ and gastric $(n=$ 1 ) origin. PR lasted a median of 24 months $(95 \%$ confidence interval: 17.4-30.5). Stable disease was observed in 29 patients (39\%):17 of pancreatic, one of colonic, five of intestinal, three of bronchial, two of unknown primary and one of thymic origin. Stable disease lasted a median of 13 months (95 \% CI: 11.3-14.8). Disease progression occurred in 26 patients (35\%).

The separate analysis of NENs of pancreatic origin demonstrated a partial remission in $32 \%(n=16 / 50)$ with a median progression free survival of 27 months (95 \% CI: 23.1-30.9). Stable disease was found in 17 patients (34\%) who had a median progression free survival of 18 months (95\% CI: 13.7-22.3). Progressive disease occurred in 17 patients (32\%) with pancreatic NEN. As shown in Fig. 1, overall progression free survival was 10 months for pancreatic and 6 months for all non pancreatic NETs, this difference, however, was not statistically significant $(p=0.65)$.

A univariate analysis of potential prognostic markers for PFS revealed that only radiologic and biochemical response to DTIC were significant prognostic markers. Patients with G1 tumors tended to have a longer PFS compared to patients with G2 tumors $(p=0.058)$. Differences in sex, age, functional activity, site of primary and number of pretreatments were not statistically significant (Table 2).

\section{Toxicity}

Chemotherapy related adverse effects were documented in 43 patients of the entire study cohort $(57.3 \%)$ but

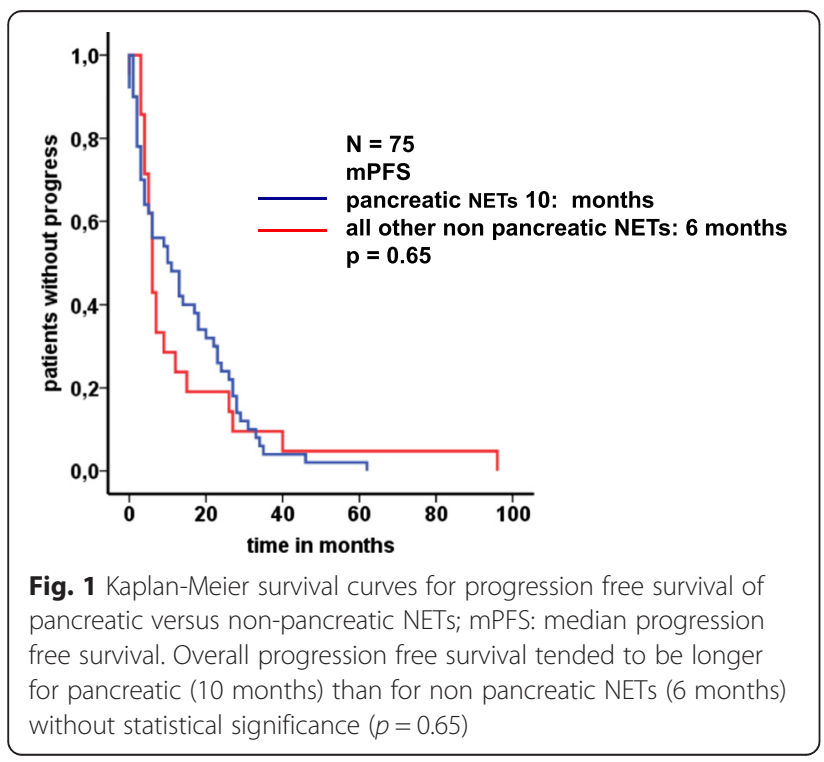

Table 2 Univariate analysis of possible prognostic factors for progression free survival

\begin{tabular}{|c|c|c|}
\hline Variable & Hazard ratio $(95 \% \mathrm{Cl})$ & $P$ value \\
\hline \multicolumn{3}{|l|}{ Sex } \\
\hline $\begin{array}{l}\text { Female } \\
\text { Male }\end{array}$ & $\begin{array}{l}1 \\
1.2(0.8-1.9)\end{array}$ & 0.46 \\
\hline \multicolumn{3}{|l|}{ Diagnosis } \\
\hline $\begin{array}{l}\text { FNA } \\
\text { FA }\end{array}$ & $\begin{array}{l}1 \\
0.9(0.5-1.6)\end{array}$ & 0.64 \\
\hline \multicolumn{3}{|l|}{ Age start CTx } \\
\hline $\begin{array}{l}<60 \\
\geq 60\end{array}$ & $\begin{array}{l}1 \\
1.1(0.7-1.7)\end{array}$ & 0.73 \\
\hline \multicolumn{3}{|l|}{ Grading } \\
\hline $\begin{array}{l}\text { G1 } \\
\text { G2 }\end{array}$ & $\begin{array}{l}1 \\
1.7(0.98-2.9)\end{array}$ & 0.058 \\
\hline \multicolumn{3}{|l|}{ Ki-67 } \\
\hline $\begin{array}{l}\leq 10 \% \\
>10 \%\end{array}$ & $\begin{array}{l}1 \\
1.1(0.6-2.1)\end{array}$ & 0.76 \\
\hline \multicolumn{3}{|l|}{ Metastases } \\
\hline $\begin{array}{l}\text { Liver only } \\
\text { Liver and others }\end{array}$ & $\begin{array}{l}1 \\
1.0(0.7-1.6)\end{array}$ & 0.91 \\
\hline \multicolumn{3}{|l|}{ Response (RECIST) } \\
\hline $\begin{array}{l}\text { No } \\
\text { Yes }\end{array}$ & $\begin{array}{l}1 \\
0.3(0.18-0.56)\end{array}$ & $0.000^{*}$ \\
\hline \multicolumn{3}{|l|}{ Biochemical response } \\
\hline $\begin{array}{l}\text { No } \\
\text { Yes }\end{array}$ & $\begin{array}{l}1 \\
0.5(0.3-0.9)\end{array}$ & $0.019^{*}$ \\
\hline \multicolumn{3}{|l|}{ Primary } \\
\hline $\begin{array}{l}\text { Non-pancreatic } \\
\text { Pancreatic }\end{array}$ & $\begin{array}{l}1 \\
0.8(0.5-1.4)\end{array}$ & 0.51 \\
\hline \multicolumn{3}{|l|}{ Prior therapy } \\
\hline $\begin{array}{l}\text { No } C T x \\
\text { CTx }\end{array}$ & $\begin{array}{l}1 \\
0.8(0.4-1.5)\end{array}$ & 0.41 \\
\hline \multicolumn{3}{|l|}{ Prior therapy } \\
\hline $\begin{array}{l}\text { SSA/IFN } \\
\text { Others }\end{array}$ & $\begin{array}{l}1 \\
0.9(0.5-1.4)\end{array}$ & 0.61 \\
\hline
\end{tabular}

${ }^{*}$ significant differences; $\mathrm{Cl}$ confidence interval, FNA functional non active, $F A$ functional active, CTx chemotherapy, SSA somatostatin analogue, IFN interferon

were usually mild with only one case of grade 3 toxicity (vomiting, $1.3 \%$ ). The most common side effects were nausea $(n=23(31 \%))$ and vomiting $(n=13 \quad(17 \%))$. Transient elevations of liver enzymes were noted in 12 patients (15\%). Mild hematotoxicity was observed in eight patients $(10.7 \%)$. Other side effects were diarrhea $(15 \%)$, fatigue (5\%) and weight loss (3\%). Only two patients stopped DTIC due to intolerable side effects. Detailed information on chemotherapy related adverse effects is given in Table 3.

\section{Discussion}

Streptozocin based chemotherapies have been the mainstay of treatment of metastatic pNET since they were 
Table 3 Toxicity

\begin{tabular}{lccccc}
\hline Side effects & $\mathrm{n}$ & $\%$ & Grade 1 & Grade 2 & Grade 3 \\
\hline Hematologic & & & & & \\
$\quad$ Anemia & 4 & 5 & 4 & - & - \\
Leukopenia & 6 & 8 & 5 & 1 & - \\
$\quad$ Thrombocytopenia & 2 & 3 & - & 2 & - \\
Gastrointestinal & & & & & \\
$\quad$ Nausea & 23 & 31 & 22 & 1 & - \\
$\quad$ Vomiting & 13 & 17 & 10 & 2 & 1 \\
$\quad$ Diarrhea & 11 & 15 & 7 & 4 & - \\
Liver & & & & & - \\
$\quad$ Elevated liver enzymes & 12 & 15 & 11 & 1 & - \\
Other & & & & & - \\
$\quad$ Fatigue & 4 & 5 & 4 & - & - \\
$\quad$ Weight loss & 2 & 3 & 2 & - & - \\
\hline
\end{tabular}

first described by Moertel in 1980 with response rates up to $69 \%$ [8]. These report rates have been questioned by others [9] because of partial reliance on nonmorphologic response criteria including clinical and biochemical assessments. Nevertheless, the efficacy of streptozocin (STZ) based therapies has been confirmed by several groups [10-16] with documented response rates of 30$40 \%$ according to radiologic criteria. Thus, STZ-based therapies have been recommended for the treatment of metastatic pNET by all international guidelines. However, disadvantages of the STZ based protocols include the need for a 5 days course and the risk of nephrotoxicity ( $9 \%$ in the Moertel group) that could preclude potential second line therapies such as PRRT.

In this study, we report on the efficacy and tolerability of low dose dacarbazine in a large single center cohort. Dacarbazine (DTIC) is a well known agent that has safely and efficiently been used for the treatment of malignant melanomas and glioblastomas for many years. In NEN patients combination treatments of low doses of DTIC (200 or $250 \mathrm{mg} / \mathrm{m}^{2}$ ) with 5-FU and epirubicin resulted in response rates between 18 and $44 \%$ [17-20]. In addition, some older studies used DTIC $250 \mathrm{mg} / \mathrm{m}^{2}$ in a 5 day treatment schedule [21-23], such as Altimari and coworkers who demonstrated response rates of $50 \%$ in a small series of 14 patients.

Evidence for the efficacy of single dose DTIC monotherapy is limited. Bukowski and coworkers investigated the use of DTIC in a series of 56 patients with NENs of various primaries at a dose of 650 and $850 \mathrm{mg} / \mathrm{m}^{2}$ showing response rates of $20 \%$ for the $850 \mathrm{mg} / \mathrm{m}^{2}$ dose and $13 \%$ for the $650 \mathrm{mg} / \mathrm{m}^{2}$ dose [24]. Ramanathan and coworkers reported a response rate of $33 \%$ in 50 patients with pancreatic NEN who received DTIC at a dose of $850 \mathrm{mg} / \mathrm{m}^{2}$. The response rate was higher in treatment naïve patients
(50\%) compared to pretreated patients (14\%) [6]. Sun et al. observed a response rate of only $8 \%$ with DTIC (250 mg/m $\mathrm{m}^{2} \mathrm{~d} 1$-d5, every 4 weeks) after failure to STZ/5FU or STZ/Doxorubicin chemotherapy in patients with advanced neuroendocrine tumors mainly of extrapancreatic origin [7]. The interpretation of the results needs some caution, since the cohorts show major differences. In all but one study NENs of all origins were included which explains some of the lower response rates, since chemotherapy is mostly effective in pNETs but not in midgut tumors, an observation as well confirmed in our study. Furthermore the studies differ in the number of patients that were pretreated, the number of pretreatments and other prognostic factors that could have influenced the treatment outcome like grading or tumor load. However, the majority of studies suggest that DTIC-based chemotherapy is active in well-differentiated NENs, though questions remain about the dosing schedule achieving the highest efficacy at an acceptable toxicity.

In accordance with these previously reported results we could clearly demonstrate in our retrospective study that DTIC chemotherapy is effective in 75 patients with well differentiated NEN, which represents the biggest series reported so far. $66 \%$ of the patients had a benefit from DTIC chemotherapy documented by an overall partial remission (PR) in $27 \%$ and disease stabilization (SD) in $39 \%$ of the patients. As expected, efficacy varied depending on the primary site of the tumor. It has to be stressed that in our series all but four patients received DTIC after disease progression to at least one prior treatment which renders the response rate even more remarkable. Median PFS in pNEN was 10 months and thus comparable to the results of everolimus and sunitinib in progressive pNET patients. Remarkably, in patients with benefit we observed sustained response or stabilization periods, lasting a median of 27 months in patients with PR and 18 months for patients with SD. These results are comparable or only slightly inferior to those achieved with the established streptozocin based protocols.

Efficacy in small bowel NENs which are known to be less chemosensitive was low (PR $9 \%$ ), whereas four of six bronchus carcinoids benefited from DTIC therapy (PR $33 \%$, SD $33 \%$ ), suggesting that this regimen may also be effective in other foregut NENs. In the same way Ekeblad and coworkers included 13 bronchial carcinoids in their study using temozolomide and found a response rate of $31 \%$ [25]. Since the number of patients with NEN of this origin was low in both series, further studies are needed to clarify the efficacy of temozolomide and dacarbazine for bronchial NEN.

In contrast to previous reports primarily with more dose intense DTIC schedules in our study DTIC demonstrated a good safety profile. While in our study side 
effects were common and were documented in $57 \%$ of the patients, they were usually mild and transient. Only two patients stopped DTIC due to side effects. Dose adjustments were necessary in only three other cases. Notably, in contrast to former studies our patients did not experience grade 3 and 4 hematotoxicity. For instance, Ramanathan et al. reported $30 \%$ grade 3 and 4 toxicities including $10 \%$ grade 4 hematotoxicity [6] probably due to the use of a higher dose of DTIC of $850 \mathrm{mg} / \mathrm{m}^{2}$.

Temozolomide is closely related to dacarbazine and shares the same active metabolite. It is available in an oral formulation, crosses the blood-brain barrier and has recently been advocated as effective, well tolerated and convenient chemotherapy for patients with NEN. Temozolomide as a single therapeutic agent has been studied by Maire and coworkers in 21 mostly pretreated NEN patients [26]. They showed a relative low response rate of $5 \%$, but a disease stabilization in $81 \%$. The Uppsala group reported comparable results using temozolomide monotherapy in patients with advanced NEN $(n=36)$ achieving a response rate of $14 \%$ and a stable disease rate of $53 \%$ [25]. Although comparisons between these studies are always very limited due to the fact that patient characteristics differ significantly, in our study DTIC does not appear to be inferior to the results reported for temozolomide monotherapy.

In combination with capecitabine, temozolomide has shown a high response rate of $70 \%$ in one small retrospective study of pancreatic NEN [3] suggesting synergistic effects between both drugs. However, all the patients in this study were chemotherapy naive. Temozolomide in combination with capecitabine therefore seems to be a promising alternative to streptozocin based therapy of metastasized pancreatic NENs. A randomized prospective trial comparing both regimens is needed to determine which is the most effective regimen that should be used in first line treatment. Most temozolomide +/- capecitabine studies reported more severe side effects, in particular grade 3 hematologic toxicity (10-15 \%) than observed in our trial. Authors have also reported a considerable incidence of opportunistic infections (10\%) related to this therapy which has rarely been reported in DTIC monotherapy and was not observed in our series [27]. Although DTIC has the disadvantage of an intravenous application route, patient discomfort is negligible, since treatment comprises a single infusion every 4 weeks in an outpatient setting. In direct comparison of the two regimens, the cost of the drug may be of relevance, since the price of a standard 5 day temozolomide course in Germany is approximately 14 times higher than the price of a single DTIC infusion. Since dacarbazine in our experience shows similar efficacy as temozolomide, we have just started to evaluate the efficacy and safety of combining dacarbazine with capecitabine.
Temozolomide has as well been used as second line chemotherapy in a small series of G3 NEN and demonstrated a response rate of $33 \%$ [28]. Response rates were higher in tumors with Ki $67<60 \%$ and in patients with positive somatostatin receptor imaging, indicating that these tumours had a higher degree of differentiation. Interestingly, adding capecitabine to temozolomide brought no additional benefit in this small series. In the same way, Olsen et al. found a response rate of $38 \%$ in 25 patients with NECs using temozolomide alone [29]. Temozolomide may therefore be a therapeutic alternative in NEC G3 tumors with a Ki 67 index between 21 and $60 \%$ which are not as aggressive as undifferentiated small cell G3 neuroendocrine cancers and often do not respond to standard cisplatin/etoposide therapy.

Apart from conventional chemotherapy the use of everolimus and sunitinib has provided novel and effective treatments for pNETs. However, the response rates achieved with these new agents overall are lower than with conventional chemotherapy and side effects can be considerable. The combination of temozolomide and everolimus has recently shown to be active in pNETs, with a response rate of $40 \%$, which is not superior to the results obtained with temozolomide plus capecitabine [30].

First promising results have as well been reported for the combination of temozolomide/capecitabine and peptide receptor radiotherapy (PRRT) in pNET with response rates of $82 \%$ [31].

Thus, conventional chemotherapy with drugs such as dacarbazine, temozolomide and/or capecitabine shows strong activity in pNET and should be included in current treatment algorithms. They may also represent the backbone for trials of novel combination therapies.

\section{Conclusions}

In summary, treatment with low dose $\left(650 \mathrm{mg} / \mathrm{m}^{2}\right)$ dacarbazine monotherapy demonstrated efficacy in progressive well differentiated NEN patients. Toxicity rates are considerably lower than those observed with high dose DTIC and combination treatments including streptozocin based regimens. Thus, low dose dacarbazine may be a therapeutic alternative for patients with well differentiated pancreatic NETs, in particular as second-line therapy. Future prospective randomized trials are necessary to further evaluate its role in patients with progressive welldifferentiated NENs of pancreatic and lung origin.

\section{Additional file}

Additional file 1: Table S1. Summary of pretreatments of our patient cohort: 25 patients received one prior treatment, 33 patients received two prior treatments, six patients received three prior treatments, two patients four prior treatments and one patient five prior treatments before starting dacarbazine. (DOCX 18 kb) 


\section{Abbreviations}

5FU, 5 flourouracil; CgA, chromogranin A; CT, computed tomography; Ctx, chemotherapy; DTIC, dacarbazine; ENETS, European Neuroendocrine Tumour Society; FA, functional active; IFN, interferon; mPFS, median progression free survival; MRI, magnetic resonance imaging; NEC, neuroendocrine carcinoma; NEN, neuroendocrine neoplasia; NET, neuroendocrine tumor; PFS, progression free survival; pNET, pancreatic neuroendocrine tumor; PR, partial remission; PRRT, peptide receptor radiotherapy; SD, stable disease; SSA, somatostatin analogue; STZ, streptozocin

\section{Acknowledgements}

We thank Iris Lenser, Maik Hahmann and Svenja Diehl for database management.

\section{Funding}

There was no funding of this retrospective study.

\section{Availability of data and materials}

The dataset supporting the conclusions of this article is available on request by contacting the authors.

\section{Authors' contributions}

DM contributed to conception and design of the study and drafted the manuscript. SK performed statistical analysis and participated in data interpretation and manuscript review. MM carried out the acquisition of data. AR was involved in conception, data interpretation and critical review of manuscript draft. TG contributed to manuscript writing and review. All authors read and approved the final manuscript.

\section{Competing interests}

The authors declare that they have no competing interests.

\section{Consent for publication}

Individual person's data are not shown, not applicable.

\section{Ethics approval and consent to participate}

This study was conducted in accordance with the Declaration of Helsinki. Collection, storage, and evaluation of patient-related information in our NEN database were performed with the approval of the local ethics committee at the University of Marburg and after obtaining the patients informed consent. Written informed consent for the treatment was obtained. The statement of the local ethics committee was that a formal approval for collection and analysis of data arising from the routine clinical evaluation within the own hospital was not required.

\section{Author details}

${ }^{1}$ Department of Gastroenterology, University Hospital Marburg, Baldinger Strasse, D35043 Marburg, Germany. ²Department of Gastroenterology, University of Halle, Ernst-Grube-Straße 40, D 06120 Halle, Germany.

\section{Received: 28 April 2016 Accepted: 28 July 2016}

\section{Published online: 18 August 2016}

\section{References}

1. Arnold R, Rinke A, Schmidt C, Hofbauer L. Endocrine tumours of the gastrointestinal tract: Chemotherapy. Best Pract Res Clin Gastroenterol. 2005; 19(4):649-56.

2. Pavel M, Baudin E, Couvelard A, Krenning E, Oberg K, Steinmuller T, Anlauf M, Wiedenmann B, Salazar R, Barcelona Consensus Conference p. ENETS Consensus Guidelines for the management of patients with liver and other distant metastases from neuroendocrine neoplasms of foregut, midgut, hindgut, and unknown primary. Neuroendocrinology. 2012;95(2): 157-76.

3. Strosberg JR, Fine RL, Choi J, Nasir A, Coppola D, Chen DT, Helm J, Kvols L. First-line chemotherapy with capecitabine and temozolomide in patients with metastatic pancreatic endocrine carcinomas. Cancer. 2011;117(2): 268-75.

4. Duncan LA, Marynick SP. Glucagonoma and dacarbazine. Ann Intern Med. 1982;97(6):930

5. van der Loos TL, Lambrecht ER, Lambers JC. Successful treatment of glucagonoma-related necrolytic migratory erythema with dacarbazine. J Am Acad Dermatol. 1987;16(2 Pt 2):468-72.
6. Ramanathan RK, Cnaan A, Hahn RG, Carbone PP, Haller DG. Phase II trial of dacarbazine (DTIC) in advanced pancreatic islet cell carcinoma. Study of the Eastern Cooperative Oncology Group-E6282. Ann Oncol. 2001;12(8): 1139-43.

7. Sun W, Lipsitz S, Catalano P, Mailliard JA, Haller DG, Eastern Cooperative Oncology G. Phase II/III study of doxorubicin with fluorouracil compared with streptozocin with fluorouracil or dacarbazine in the treatment of advanced carcinoid tumors: Eastern Cooperative Oncology Group Study E1281. J Clin Oncol. 2005:23(22):4897-904.

8. Moertel CG, Hanley JA, Johnson LA. Streptozocin alone compared with streptozocin plus fluorouracil in the treatment of advanced islet-cell carcinoma. N Engl J Med. 1980;303(21):1189-94.

9. Cheng PN, Saltz LB. Failure to confirm major objective antitumor activity for streptozocin and doxorubicin in the treatment of patients with advanced islet cell carcinoma. Cancer. 1999;86(6):944-8.

10. Eriksson B, Skogseid B, Lundqvist G, Wide L, Wilander E, Oberg K. Medical treatment and long-term survival in a prospective study of 84 patients with endocrine pancreatic tumors. Cancer. 1990;65(9):1883-90.

11. Rivera E, Ajani JA. Doxorubicin, streptozocin, and 5-fluorouracil chemotherapy for patients with metastatic islet-cell carcinoma. Am J Clin Oncol. 1998:21(1):36-8.

12. Delaunoit $T$, Ducreux $M$, Boige $V$, Dromain $C$, Sabourin JC, Duvillard $P$, Schlumberger $M$, de Baere T, Rougier $P$, Ruffie $P$, et al. The doxorubicinstreptozotocin combination for the treatment of advanced welldifferentiated pancreatic endocrine carcinoma; a judicious option? Eur J Cancer. 2004:40(4):515-20.

13. Fjallskog ML, Janson ET, Falkmer UG, Vatn MH, Oberg KE, Eriksson BK. Treatment with combined streptozotocin and liposomal doxorubicin in metastatic endocrine pancreatic tumors. Neuroendocrinology. 2008;88(1): $53-8$

14. Dilz LM, Denecke T, Steffen IG, Prasad V, von Weikersthal LF, Pape UF, Wiedenmann B, Pavel M. Streptozocin/5-fluorouracil chemotherapy is associated with durable response in patients with advanced pancreatic neuroendocrine tumours. Eur J Cancer. 2015:51(10):1253-62.

15. Krug S, Boch M, Daniel H, Nimphius W, Müller D, Michl P, Rinke A, Gress TM. Streptozocin-based chemotherapy in patients with advanced neuroendocrine neoplasms - predictive and prognostic markers for treatment stratification. PLoS One. 2015;10(12), e0143822.

16. Clewemar Antonodimitrakis P, Sundin A, Wassberg C, Granberg D, Skogseid B, Eriksson B. Streptozocin and 5-FU for the treatment of pancreatic neuroendocrine tumors: efficacy, prognostic factors and toxicity. Neuroendocrinology. 2016;103(3-4):345-53.

17. Bajetta E, Ferrari L, Procopio G, Catena L, Ferrario E, Martinetti A, Di Bartolomeo M, Buzzoni R, Celio L, Vitali M, et al. Efficacy of a chemotherapy combination for the treatment of metastatic neuroendocrine tumours. Ann Oncol. 2002;13(4):614-21

18. Bajetta E, Rimassa L, Carnaghi C, Seregni E, Ferrari L, Di Bartolomeo M Regalia E, Cassata A, Procopio G, Mariani L. 5-Fluorouracil, dacarbazine, and epirubicin in the treatment of patients with neuroendocrine tumors. Cancer 1998;83(2):372-8.

19. Walter T, Bruneton D, Cassier PA, Hervieu V Pilleul F, Scoazec JY, Chayvialle JA, Lombard-Bohas C. Evaluation of the combination 5-fluorouracil, dacarbazine, and epirubicin in patients with advanced welldifferentiated neuroendocrine tumors. Clin Colorectal Cancer. 2010;9(4): 248-54.

20. Di Bartolomeo M, Bajetta E, Bochicchio AM, Carnaghi C, Somma L, Mazzaferro V, Visini M, Gebbia V, Tumolo S, Ballatore P. A phase II trial of dacarbazine, fluorouracil and epirubicin in patients with neuroendocrine tumours. A study by the Italian Trials in Medical Oncology (I.T.M.O.) Group. Ann Oncol. 1995:6(1):77-9.

21. Kessinger A, Foley JF, Lemon HM. Therapy of malignant APUD cell tumors Effectiveness of DTIC. Cancer. 1983;51(5):790-4.

22. van Hazel GA, Rubin J, Moertel CG. Treatment of metastatic carcinoid tumor with dactinomycin or dacarbazine. Cancer Treat Rep. 1983;67(6):583-5.

23. Altimari AF, Badrinath $K$, Reisel HJ, Prinz RA. DTIC therapy in patients with malignant intra-abdominal neuroendocrine tumors. Surgery. 1987;102(6): 1009-17.

24. Bukowski RM, Tangen CM, Peterson RF, Taylor SA, Rinehart JJ, Eyre HJ, Rivkin SE, Fleming TR, Macdonald JS. Phase II trial of dimethyltriazenoimidazole carboxamide in patients with metastatic carcinoid. A Southwest Oncology Group study. Cancer. 1994;73(5):1505-8. 
25. Ekeblad S, Sundin A, Janson ET, Welin S, Granberg D, Kindmark H, Dunder K, Kozlovacki G, Orlefors $\mathrm{H}$, Sigurd M, et al. Temozolomide as monotherapy is effective in treatment of advanced malignant neuroendocrine tumors. Clin Cancer Res. 2007;13(10):2986-91.

26. Maire F, Hammel P, Faivre S, Hentic O, Yapur L, Larroque B, Couvelard A, Zappa M, Raymond E, Levy P, et al. Temozolomide: a safe and effective treatment for malignant digestive endocrine tumors. Neuroendocrinology. 2009;90(1):67-72.

27. Schwarzberg AB, Stover EH, Sengupta T, Michelini A, Vincitore $M$, Baden $L R$, Kulke MH. Selective lymphopenia and opportunistic infections in neuroendocrine tumor patients receiving temozolomide. Cancer Investig. 2007;25(4):249-55.

28. Welin S, Sorbye H, Sebjornsen S, Knappskog S, Busch C, Oberg K. Clinical effect of temozolomide-based chemotherapy in poorly differentiated endocrine carcinoma after progression on first-line chemotherapy. Cancer. 2011;117(20):4617-22.

29. Olsen $\mathrm{IH}$, Sorensen JB, Federspiel B, Kjaer A, Hansen CP, Knigge U, Langer SW. Temozolomide as second or third line treatment of patients with neuroendocrine carcinomas. Sci World J. 2012;2012:170496.

30. Chan JA, Blaszkowsky L, Stuart K, Zhu AX, Allen J, Wadlow R, Ryan DP, Meyerhardt J, Gonzalez M, Regan E, et al. A prospective, phase $1 / 2$ study of everolimus and temozolomide in patients with advanced pancreatic neuroendocrine tumor. Cancer. 2013;119(17):3212-8.

31. Claringbold PG, Price RA, Turner JH. Phase I-II study of radiopeptide 177Lu-octreotate in combination with capecitabine and temozolomide in advanced low-grade neuroendocrine tumors. Cancer Biother Radiopharm. 2012;27(9):561-9.

\section{Submit your next manuscript to BioMed Central and we will help you at every step:}

- We accept pre-submission inquiries

- Our selector tool helps you to find the most relevant journal

- We provide round the clock customer support

- Convenient online submission

- Thorough peer review

- Inclusion in PubMed and all major indexing services

- Maximum visibility for your research

Submit your manuscript at www.biomedcentral.com/submit 